\title{
A Model for Conceptual Processing of Naturalistic Scenes
}

\author{
AURA HANNA Virginia Commonwealth University \\ GEOFFREY LOFTUS University of Washington
}

\begin{abstract}
Are there fundamental differences in the way in which a list of pictures and a list of words are processed? We report three experiments that examine serial position effects for rapidly-presented naturalistic scenes. The experiments provide a basis for comparison with the U-shaped serial position curve and list-length effect which typically result from verbal learning experiments. In contrast to the U-shaped verbal serial position function, our results show a flat function at the beginning serial positions and a recency effect which is small and limited to the last serial position. There is a set-size effect. Results suggest that the processing leading to a memory representation for visual stimuli such as pictures and linguistic stimuli such as words is qualitatively dissimilar. The findings can be accounted for by a serial processing model whose main parameter is the probability that the subject will switch attention from one picture to the next.
\end{abstract}

Résumé Y a-t-il des différences fondamentales dans la façon dont sont traitées une liste d'images et une liste de mots? À l'aide de trois expériences, nous avons examiné les effets liés à la position sérielle d'images illustrant des scènes naturelles, présentées rapidement. Ces expériences offrent une base de comparaison avec la courbe de positions sérielles en forme de $\mathbf{U}$ et l'effet de la longueur de la liste, qui caractérisent les expériences sur l'apprentissage verbal. Contrairement à la courbe en $U$ observée dans le cas des positions sérielles verbales, nos résultats révèlent une fonction stable au début des positions sérielles et un faible effet de récence limité à la demière position sérielle. La taille de la série de stimuli visuels influe sur la performance. Les résultats obtenus nous portent à croire que le traitement de stimuli visuels tels les images et le traitement de stimuli linguistiques comme les mots, qui conduisent à une représentation en mémoire, differrent sur le plan qualitatif. Un modèle de traitement sériel dont le principal paramètre est la probabilité que le sujet prête attention aux images présentées tour à tour peut rendre compte des observations.

When a person views a picture or a word, the result is a memory representation that depends on how the picture or word is processed. In this article, we 
seek to understand differences between processing of pictures and words. We present a model of picture-processing which is a simplified version of an earlier model (Loftus, Hanna, \& Lester, 1988). The model's main parameter is the probability that the subject will switch attention from one serially-presented picture to the next.

\section{PERCEPTUAL AND CONCEPTUAL PROCESSING}

Theorists (e.g., Intraub, 1984; Loftus \& Ginn, 1984; Potter, 1976) have distinguished two kinds of encoding processes that operate on visual stimuli. Perceptual processes are defined as processes that operate on, and thus require the presence of, a stimulus or visible persistence of that stimulus. Conceptual processes are defined as those that do not require a stimulus or its visible persistence. Conceptual processes can operate while the stimulus is physically present, while there is visible persistence of the stimulus, and after any visible persistence has ceased.

\section{Perceptual and Conceptual Masking}

A visual stimulus presented close in time to a target stimulus is called a mask and any target performance decrement that results is a masking effect. A distinction can be made between perceptual and conceptual masks. Perceptual masks interrupt or interference with perceptual processing. Conceptual masks interrupt or interfere with conceptual processing. A mask following target stimulus offset by $300 \mathrm{~ms}$ or more exerts only a conceptual masking effect whereas a mask following target stimulus onset by $50 \mathrm{~ms}$ or less exerts only a perceptual masking effect (Hines \& Smith, 1977, Loftus \& Ginn, 1984). In this paper we are interested in conceptual processing and conceptual masking; hence all our interstimulus intervals (ISIs) are at least $300 \mathrm{~ms}$.

\section{Serial Position Effects}

The serial position effect is the effect of item presentation position on memory performance. Examination of serial position effects provides a means of investigating conceptual masking. Consider a sequence of successivelypresented items to be remembered. Each item following the first is a potential conceptual mask for its predecessors. Conceptual masking effects will be revealed as decrements in memory performance at specific serial positions.

Serial Position Effects for Verbal Stimuli. Serial position effects have typically been measured in verbal learning experiments using free recall as the measure of memory performance. When performance is plotted, the resulting curve is U-shaped. The verbal serial position curve has four major characteristics. Usually the first two or three items are better remembered: this is the primacy effect. Similarly, the last two or three items are better remembered: this is the recency effect. At the middle positions, the performance curve typically shows a relatively flat asymptote. 
The primacy effect shown for free recall of verbal stimuli is usually attributed to differential encoding of the first items (Crowder, 1976). It can be reduced or eliminated by changing the method of presentation from single words to word pairs (Murdock, 1964; Raaijmakers \& Shiffrin, 1981), by preventing rehearsal (Glenberg, 1984), or by instructing subjects to rehearse each item only for its duration (Raffel, 1936). Results using recognition as the verbal memory measure are more mixed than those using recall: some show a multiple-item primacy effect (Cohen, 1970; Loftus, 1974), some show a single-item effect Cohen, 1970), others show no effect (Bjork \& Whitten, 1974). Considered together, results using recall and recognition measures suggest that the primacy effect in verbal memory is produced at encoding, and that it does not result from performance measures differences which would more likely be exhibited at retrieval.

The recency effect shown for verbal recall is explained as due to temporal discriminability at retrieval, i.e., items entered into a memory store later are easier to retrieve than those entered earlier (Crowder, 1976). Memory performance is a function of ease of retrievability. Some experiments have shown the large, multiple-item recency effects for word recognition (Loftus, 1974) which are typical of word recall. However, others have shown a small or null effect (Bjork \& Whitten, 1974; Cohen, 1970; Goodwin, 1976; Rundus, Loftus, \& Atkinson, 1970).

Serial Position Effects for Visual Stimuli. In contrast to the many experiments examining serial position effects for verbal material, there have been few experiments examining serial position effects for visual stimuli such as pictures, forms, patterns, and objects, and there is little or no theory.

One study (Loftus, 1974) makes a direct comparison between visual and verbal stimuli. Loftus tested recognition memory performance for words and randomly-generated forms. For word recognition, he found multiple-item primacy and recency effects. The shape of the serial position curve was quite different for form recognition, however. It showed no primacy effect and a recency effect limited to one-item.

The primacy effect is usually not shown for visual stimuli. For recall, it is more often absent (Christie \& Phillips, 1979; Shiffrin, 1973) than present (Gillund \& Shiffrin, 1981). For recognition, it is typically absent or negative (Intraub \& Nicklos, 1981; Loftus, 1974; Phillips \& Christie, 1977; Potter \& Levy, 1969; Shaffer \& Shiffrin, 1972).

The recency effect is also different from that shown for verbal memory. For recall measures, it is absent (Gillund \& Shiffrin, 1981; Shiffrin, 1973) or a one-item effect (Christie \& Phillips, 1979). For recognition measures, a one-item recency effect is typical (Atkinson, Hansen, \& Bernbach, 1964; Intraub \& Nicklos, 1981; Loftus, 1974; Phillips \& Christie, 1977; Potter, 1976; Potter \& Levy, 1969).

Thus, those experiments examining serial position effects for pictures or 
other visual stimuli typically show a null or negative primacy effect and a one-item recency effect. This contrasts with the multiple-item primacy and recency effects typical of verbal serial position curves.

\section{Set-Size Effect}

In verbal memory experiments, the shorter the list, the higher is the ratio of primacy and recency to asymptote and the better is memory performance: this is the list-length effect. For visual stimuli, it is called the set-size effect. List-length effects are typical for verbal stimuli (Gillund \& Shiffrin, 1981; Shiffrin, 1973). Picture memory performance also typically shows a set-size effect, although it is often not as large as that shown for verbal memory performance (Gillund \& Shiffrin, 1981; Intraub \& Nicklos; 1981; Murdock, 1962; Shiffrin, 1973).

In the following section we present a conceptual processing model which is a revision of a model developed by Loftus et al. (1988). The model's primary assumption is that conceptual processing is a serial process. This assumption leads to predictions about the shape of the serial position curves. We then present experiments which examine serial position effects in the context of conceptual processing and conceptual masking. Finally, we assess the model's validity in accounting for the results.

\section{A CONCEPTUAL PROCESSING MODEL}

Loftus et al. (1988) described a conceptual processing model that accounted for data gathered in a series of conceptual-masking experiments involving manipulation of conceptual mask duration, mask content, and whether the mask was itself perceptually masked. The model predicted a primacy effect, a recency effect, and a set-size effect. A quantitative form of the model was used to fit data from two picture memory experiments reported by Intraub and Nicklos (1981). It effectively predicted the set-size and the recency effects. However, contrary to the model's prediction, Intraub and Nicklos found no primacy effect. The revised model does not predict a primacy effect and we have set some of the parameters based on previous estimates. Thus the number of free parameters in the revised model is reduced. In two of the experiments, there is only one free parameter; in one experiment, two parameters are free.

In this section, we describe the qualitative assumptions and the parameters, then specify the model's predictions.

\section{Qualitative Assumptions}

The model has five qualitative assumptions. We describe them within the context of a paradigm wherein subjects are presented with a sequence of complex, unrelated naturalistic scenes to be remernbered. In this paradigm, each scene following the first is a potential conceptual mask for its prede- 
cessors. A relatively long ISI ensures that only conceptual masking can occur. The assumptions are as follows.

1. Conceptual processing operates on perceptual information. Conceptual processing requires some perceptual information to act upon thus, for each scene, it begins after the onset of perceptual processing. Conceptual processing of a scene can continue concurrently with perceptual processing of the subsequent scene.

2. The initiation of conceptual processing is probabilistic. For conceptual processing to occur, a scene must capture attention. The initiation of conceptual processing is determined by the attention demands of the various scenes in the sequence. In the model, we assume that the probability ranges from zero to 1.0 that any scene will capture attention.

3. Conceptual processing is a serial process. If conceptual processing begins on one scene, it ceases on the preceding scene. This attention switch from a preceding to a succeeding scene constitutes conceptual masking of the preceding scene. The serial processing assumption leads to predictions for the shape of the serial position curve which differ from the U-shaped verbal serial position curve.

4. Conceptual processing results in a memory representation. The quality of the memory representation is determined by the amount of acquired perceptual information on which conceptual processing operated and by the amount of conceptual processing time.

5. Memory performance is determined by the quality of the memory representation. Observed performance is a function of the amount of acquired perceptual information and the amount of conceptual processing time.

\section{Quantitative Model Parameters}

The model is a serial processing model with three parameters, the attention-switching probability, $k$, the perceptual information acquisition rate, $c$, and the conceptual processing rate, $s$, which are described in more detail below. In order to make quantitative predictions, it is necessary to instantiate the model as a quantitative model. The quantitative equations are contained in the Appendix. The quantitative contribution of the $c$ parameter is shown in Equations 1 and 1a. The $s$ parameter is embodied in Equation 2. The attention-switching parameter, $k$, is contained in Equations 3 and 4 which show the overall probability of learning.

1. Perceptual information acquisition rate: $c$. The $c$ parameter determines the rate at which perceptual information is acquired. The higher is $c$, the faster is information acquisition. Higher $c$ values would be expected, for example, with high-relative to low-luminance stimuli or with more alert relative to less alert observers. The value of $c$ was estimated to be 3.4 by Loftus et al. (1988) using similar stimuli. We use this estimate to fit the data to the model for the first two experiments. In the third experiment, wherein luminance was 
reduced, we permit the $c$ parameter to vary.

2. Conceptual processing rate: $s$. The $s$ parameter determines the rate at which conceptual processing occurs. Higher $s$ values imply a more completely encoded stimulus at any given stimulus onset asynchrony (SOA). The value of $s$ would be expected to be higher for simple than for complex pictures, for example, because simple pictures are intrinsically easier to encode. Loftus et al. (1988) estimated the value of $s$ to be 2.14 using similar stimuli. We use this estimate to fit the experimental data to the model.

3. Attention switching probability: $k$. The $k$ parameter specifies the probability that a succeeding item will capture attention from its predecessor. The recency effect is partly a function of $k$. As the value of $k$ increases, the probability that the last picture in the set will capture attention increases and, given that it has captured attention, conceptual processing of it can continue into the post-set interval. Thus the predicted recency effect increases with the value of $k$.

\section{Model Predictions}

In our model, the shape of the serial position curve is determined by the attention-switching probability, $k$. The model makes specific predictions about the effects of serial position, as follows.

1. Assuming $k>0$, the first and middle pictures in a sequence are approximately equally well-remembered. Each picture has an equal probability of capturing attention. In contrast to typical verbal serial position curves, no primacy effect is predicted.

2. Assuming $k>0$, the last item in a sequence is remembered better than the middle items; there is nothing to capture attention from it and therefore no conceptual masking effect. Thus a one-item recency effect is predicted.

3. The ratio of the last item to middle items will be higher for sets containing fewer items. Accordingly, memory performance should be a function of set size, smaller sets resulting in higher performance. Because the predicted recency effect is of only one item, as set size increases, the set-size effect will decrease.

In summary, the model predicts a relatively flat function at the beginning and middle positions which is independent of set size, a recency effect, and a set-size effect.

\section{Experiments}

In this section, we report three experiments designed to generate serial position curves for naturalistic scenes. We vary set size, stimulus duration, and stimulus luminance. We compare the experimental data to that predicted by our conceptual processing model and to verbal serial position curves.

In the first two experiments, only one parameter varies in the model, the attention switching probability, $k$. Two other parameters, $s$ and $c$, are set on 
the basis of previous experimental results. In the last experiment, the perceptual information acquisition rate, $c$, is also allowed to vary.

\section{GENERAL METHOD}

The paradigm that we use is patterned after that described by Intraub and Nicklos (1981). On each trial, a sequence of pictures was shown. Within trials, pictures were arranged in sets of varying size; however, the total number of pictures shown and the total study time for each trial was constant. Holding total trial time constant permits us to assess set-size effects independent of amount of available processing time.

As much of the methodology is identical over the four experiments, we first define our terms and present methodological features common to all experiments. In the methodology sections of individual experiments, we describe details and unique features.

\section{Subjects}

All subjects were University of Washington undergraduates who participated for course credit. They were run in groups of $5-8$ subjects per group.

\section{Materials}

The stimuli were naturalistic photographs prepared as $35-\mathrm{mm}$ colour slides which were originally taken from various "vacation picture" collections. They depicted seascapes, landscapes, and cityscapes and included such scenes as a rural village seen from the air, sailboats on a lake, a lake surrounded by mountains, and snow-capped mountains. The slides were randomly placed into four slide trays. Either 54 or 53 slides were used from each of the first three trays to total 160 slides which were designated as targets. The fourth tray contained 80 slides which were designated as distractors. A target refers to a photograph shown in a recognition test for which the correct answer is old and a distractor refers to a photograph shown in a recognition test for which the correct answer is new. A dim adapting field was present throughout all experiments'.

\section{Apparatus}

Stimuli were displayed via one Kodak random-access projector and three Kodak standard projectors. Stimuli subtended a visual angle that ranged from 15 to 22 degrees horizontal, and from 10 to 15 degrees vertical, depending on where the subject sat. Timing was controlled by Gerbrands tachistoscopic

1 Stimulus luminances, in millilamberts, were as follows: adapting field - 0.05 ; projector on, no slide - 36.47; range of typical stimulus slide - $1.59-28.31$. 
shutters with rise and fall times of approximately $1 \mathrm{~ms}$. All display equipment was enclosed in a soundproof box. Slides were projected onto a white wall. Subjects made all responses on individual response boxes. Except for one projector, the display and response-collection equipment was controlled by an Apple II computer system.

Distractor slides were shown via a standard projector; the random access projector and two standard projectors were used to present the target slides.

\section{Design and Procedures}

The basic design was the same throughout all experiments. On each trial, a sequence of eight briefly-presented scenes was shown. Within the sequence, scenes were grouped in various ways; however, the total number of scenes shown and the total study time for each trial was constant. The specific procedures were as follows.

Study procedure. Four experimental conditions were determined by the number of sets in a trial. A trial consisted of a sequence of eight scenes sequentially displayed as one 8-scene set, two 4-scene sets, four 2-scene sets, or eight 1-scene sets. Within each set, scenes were presented rapidly; each set was followed by a post-set interval. A block consisted of four trials, each trial incorporating one of the four conditions. An experimental session consisted of five study-test blocks.

The time for each trial was typically $12,600 \mathrm{~ms}$, divided as follows. Each of the 8 scenes in a trial was shown for $50 \mathrm{~ms}$ and was followed by a $400-\mathrm{ms}$ ISI. The total display and ISI time was therefore $450 \mathrm{~ms}$ for each scene for a total of $3600 \mathrm{~ms}$ per trial. Scenes were grouped as: eight 1-scene sets, four 2-scene sets, two 4-scene sets, or one 8-scene set (continuous). Corresponding post-set intervals were: $1000 \mathrm{~ms}$ (following each of the eight, 1-scene sets), $2000 \mathrm{~ms}$ (following each of the four, 2-scene sets), $4000 \mathrm{~ms}$ (following each of the two, 4-scene sets), or $8000 \mathrm{~ms}$ (following the 8-scene set). The total interval time was therefore $8000 \mathrm{~ms}$ in all conditions. A $1000-\mathrm{ms}$ tone began each trial. A trial thus consisted of $3600 \mathrm{~ms}$ display and ISI time, $8000 \mathrm{~ms}$ of interval time, and $1000 \mathrm{~ms}$ of tone time, for a total of $12,600 \mathrm{~ms}$. The timing and sequence of events for the 4-scene set condition is depicted schematically in Figure 1.

In all experiments, subjects were informed about the timing and sequence of events that would occur and were instructed to try and remember all the pictures that they saw.

Test procedure. Memory was always tested using old/new recognition tests. The following test procedure was common to all experiments. The proportion of targets to distractors was 2:1. After each four-trial block, a recognition test was given which included the 32 target scenes from the preceding four trials along with 16 distractors. For each test stimulus, subjects were asked to respond "yes" or "no" corresponding to whether they thought they had or had 


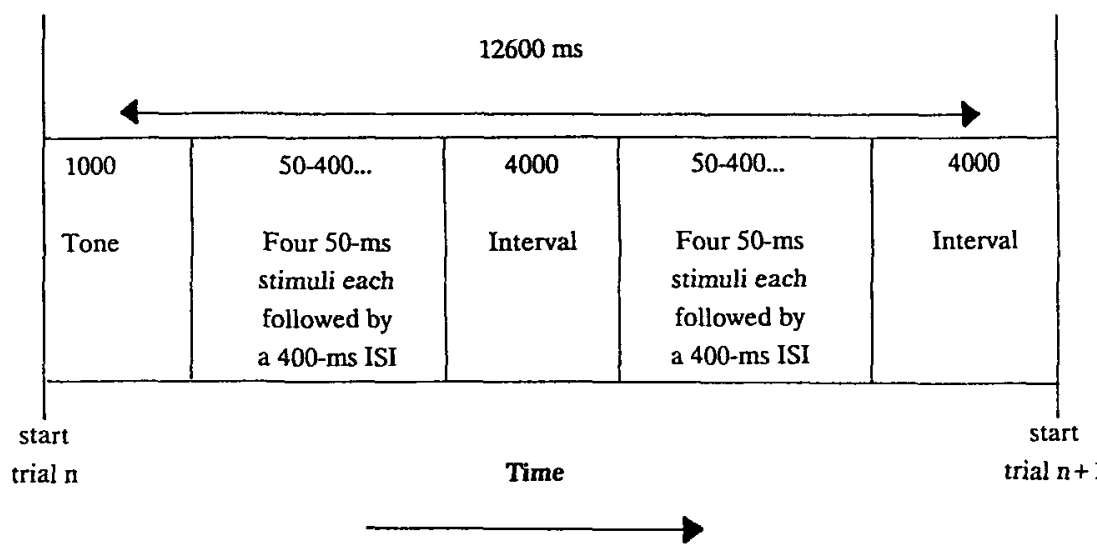

Fig. 1 Sequence of Events: Two 4-scene Sets Condition

not seen the picture in the preceding study phase. Subjects responded by pressing a key on their response boxes after they heard a 100-ms beep which occurred $600 \mathrm{~ms}$ following the test picture's onset. Each test picture remained on the screen until all subjects had responded. There was a 1500-ms interval between the last subject's response and the beginning of the next test trial during which the experimenter advanced the one projector not controlled by the computer.

Counterbalancing. There were four conditions. The order of the study conditions within a block was rotated over blocks so that a different condition began each successive block; these orders were in turn rotated so that the start order changed every four groups. For example, for groups $1-4$ the condition order was 1-2-3-4 for block 1 and 2-3-4-1 for block 2; for groups 5-8, it was 2-3-4-1 for block 1 and 3-4-1-2 for block 2 .

To achieve at least partial counterbalancing of slides and conditions, we advanced the start position of each of the target projectors forward 1 slide for every $x$ groups, $x$ being the total number of conditions. For groups $1-4$, for example, all projectors would start at slide 1 ; for groups $5-8$, all projectors would start at slide 2 . Further counterbalancing details are provided with individual experiments.

The target-distractor order at test was random for each block but was constant for all subjects. Target stimuli were shown in the order presented at study.

Fitting experimental data to model predictions. To obtain predicted values from the model, we use a grid search procedure to find the parameter values that minimized the root mean square error (RMSE) between observed and predicted values. The RMSE is interpreted as the average discrepancy between an observed and a predicted condition mean; it is in units of the original dependent variable (corrected hit probability). 


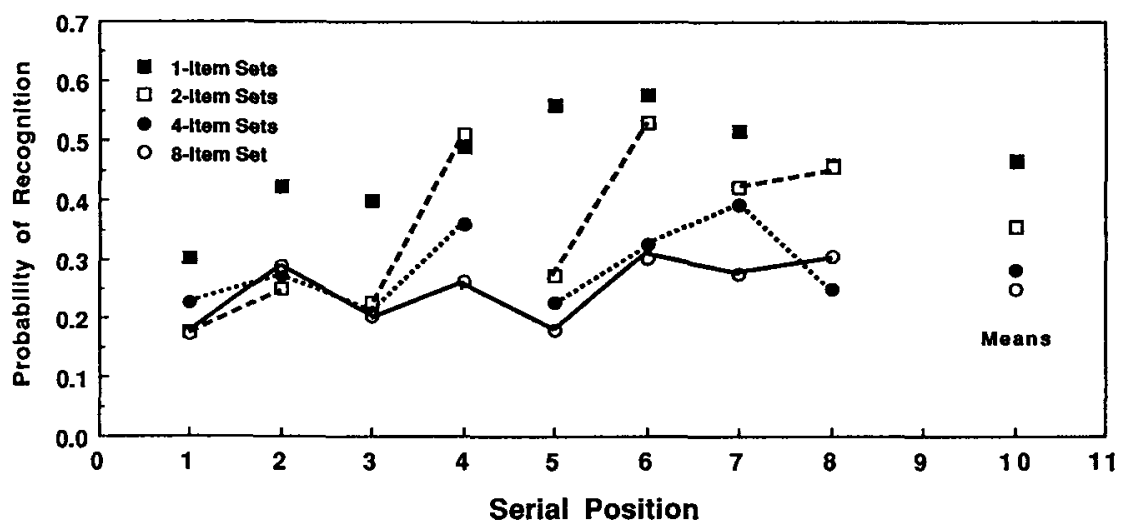

Fig. 2 Experiment 1

\section{Experiment 1}

Experiment 1 was designed to investigate conceptual masking by examining serial position and set-size effects for sequentially-presented naturalistic pictures. Set-size and recency effects predicted by our serial-processing model depend on differential processing of only the last item. Parallel processing models assume that items are processed more than one-at-a-time; therefore, any differential processing effects predicted by parallel processing models are for multiple, not single items. Thus, results showing set-size effects for only small sets or single-item recency effects confirm our model's serial processing assumption.

\section{METHOD}

Subjects. Ninety-three subjects were run in 16 groups.

Design and procedure. In Experiment 1, the target duration was $50 \mathrm{~ms}$. With 16 groups, counterbalancing procedures ensured that each slide was seen in four of the eight serial positions. All other procedures were as specified in the General Method section.

\section{RESULTS AND DISCUSSION}

The results of Experiment 1 are presented in Figure 2. Means of the four conditions are shown at the right in Figure 2.

To better enable comparison among experiments, observed and predicted values for all experiments are presented in Table 1. As can be seen in Table 1, the model does a good job of fitting the shape of the curves for Experiment 1: the RMSE between observed and predicted values was 0.054 . We evaluated the fit of the model statistically by testing the null hypothesis that the model is correct. The difference was not significant, $F(14,28)=0.068$.

The best fitting value for $k$, the attention-switching parameter, was 0.88 . This can be interpreted to mean that, on average, a scene which captured 
TABLE 1

Observed/Predicted Values

\begin{tabular}{lcccc}
\hline Exp. 1 & & & & \\
\hline Serial. Pos. & 8-Item Set & 4-Item Sets & 2-Item Sets & 1-Item Sets \\
1 & $.173 / .243$ & $.226 / .243$ & $.276 / .248$ & $.467 / .400$ \\
2 & $.289 / .243$ & $.300 / .243$ & $.436 / .352$ & \\
3 & $.203 / .243$ & $.303 / .248$ & & \\
4 & $.262 / .243$ & $.305 / .352$ & & \\
5 & $.181 / .243$ & & & \\
6 & $.304 / .243$ & & & \\
7 & $.277 / .248$ & & & \\
8 & $.306 / .352$ & & $.356 / .300$ & $.467 / .400$ \\
Mean & $.249 / .257$ & $.284 / .272$ & & \\
RMSE $=.054 ; \mathrm{k}=0.88$ & & &
\end{tabular}

Exp. 2 Increased Duration

\begin{tabular}{lcccc}
\hline Serial. Pos. & 8-Item Set & 4-Item Sets & 2-Item Sets & 1-Item Sets \\
1 & $.323 / .379$ & $.360 / .379$ & $.401 / .384$ & $.561 / .573$ \\
2 & $.393 / .379$ & $.357 / .379$ & $.572 / .504$ & \\
3 & $.381 / .379$ & $.378 / .384$ & & \\
4 & $.443 / .379$ & $.505 / .504$ & & \\
5 & $.346 / .379$ & & & \\
6 & $.357 / .379$ & & & \\
7 & $.360 / .384$ & & & \\
8 & $.458 / .504$ & & & \\
Mean & $.383 / .395$ & $.400 / .412$ & $.486 / .444$ & $.561 / .573$ \\
RMSE $=.035 ; \mathrm{k}=0.88$ & & & \\
\hline
\end{tabular}

Exp. 3 Reduced Luminance

\begin{tabular}{lcccc}
\hline Serial. Pos. & 8-Item Set & 4-Item Sets & 2-Item Sets & 1-Item Sets \\
1 & $.179 / .120$ & $.076 / .120$ & $.126 / .120$ & $.239 / .194$ \\
2 & $.110 / .120$ & $.101 / .120$ & $.232 / .192$ & \\
3 & $.055 / .120$ & $.071 / .120$ & & \\
4 & $.201 / .120$ & $.226 / .192$ & & \\
5 & $.175 / .120$ & & & \\
6 & $.117 / .120$ & & & \\
7 & $.022 / .120$ & & & \\
8 & $.127 / .192$ & & & \\
Mean & $.123 / .129$ & $.118 / .138$ & $.179 / .156$ & $.239 / .194$ \\
RMSE $=.056 ; \mathrm{k}=0.99 ; \mathrm{c}=1.44$ & & \\
\hline
\end{tabular}


attention received processing for $1 / k=1.14$ SOA units or $511 \mathrm{~ms}$. Thus, for an individual subject, all stimuli in a trial cannot be conceptually processed. The result is that the subject is unable to distinguish some studied stimuli from similar distractors.

There is a set-size effect: the smaller is the set, the higher is mean performance, $F(3,200)=22.37^{2}$. Because the ratio of the last item to other items decreases as set size increases, the model predicts a set-size effect for small but not larger sets. This prediction is confirmed. Analyses by t-test showed that there were significant differences between the 1- and 2-scene sets, $t(7)=3.21$, and between the 2 - and 4-scene sets, $t(7)=2.15, p<.05$. Larger set sizes do not show the effect: the 4- and 8-scene sets were not significantly different, $t(7)=1.27$.

In the 8- and 4-scene sets, the function is flat at the beginning positions: the first position is not significantly different from the mean of the middle positions, collapsed over sets in a trial, by Scheffé tests, $F^{\prime}(1,644)=2.81$ and $F^{\prime}(1,276)=5.66$ for the 8- and 4-scene sets respectively. However, in the 1 -scene set, there is a negative primacy effect rather than the predicted flat function; that is, memory performance for the first scene is lower than for subsequent scenes. The difference was significant by a Scheffé test against the mean of the middle six positions, $F^{\prime}(1,644)=19.18$.

The model predicts a recency effect in the 8-, 4-, and 2-scene sets and a flat function in the 1-scene set. Planned comparisons of the last position against the middle positions (excluding the first) with the data collapsed across sets in a trial indicated that there was a significant recency effect in the data only in the 2 -scene set, $F(1,176)=52.98$.

The model predicts a small increment in memory performance for the item at serial position $n-1$, as Table 1 shows. This is for the following reason. The last scene has a probability, $k=.88$, of capturing attention and a probability, $(1-k)=.12$, of not capturing attention. If the last scene does not capture attention, processing of the attended stimulus can continue. Among the previous stimuli, the probability is highest that the attended stimulus will be the second-to-last stimulus. With a $k$ value of less than unity, the model therefore predicts an increased probability of recognition for the second-to-last stimulus over the previous stimulus. This is shown quantitatively in Equation 3.

Standard errors of the mean were computed for three of the four conditions. For the 8-scene set, it was 0.022; for the 4-scene set, 0.016; for the 2-scene set, 0.010 . The small standard errors, relative to amount of variation over serial position, reflect consistency of the curve's shape over subjects ${ }^{3}$.

2 Unless otherwise indicated, reported results are significant at the 0.01 level.

3 To compute the est $\sigma_{\mathrm{M}}$ in this within-subject design, we divided the square root of the MSI (subject $x$ serial position) by the square root of $\mathbf{n}$ (Loftus \& Loftus, 1988). 
Because the four conditions were intermingled during the study phase, there is only a single false-alarm probability, which in Experiment 1 was 0.330 .

In contrast to verbal serial position curves, the curves resulting from Experiment 1 are relatively flat - there is no primacy effect and a recency effect is shown in only one condition. The set-size effect is shown for relatively small set sizes but not for larger sizes. Results are consistent with a serial processing model.

\section{Experiment 2: Increased Stimulus Duration}

With verbal stimuli, typically, presentation rate has an effect on the beginning portion of the curve but not on the recency portion (Bernbach, 1975; Glanzer \& Cunitz, 1966; Murdock, 1962). In some experiments examining processing of visual stimuli, this same pattern is shown (Hines, 1975; Phillips \& Christie, 1977) but in others the increment is shown across all serial positions (Tversky \& Sherman, 1975). In Experiment 2, we increased the stimulus duration from 50 to $150 \mathrm{~ms}$. Increasing the stimulus duration increases the amount of acquired perceptual information, and also increases the SOA which results in more conceptual processing time. Thus the model predicts an increment in the performance level across all serial positions. Experiment 2 was like Experiment 1 in all respects except for the increased stimulus duration.

\section{METHOD}

Subjects. Sixty-six subjects were run in 12 groups.

Design and procedure. In Experiment 2, the stimulus duration was increased from $50 \mathrm{~ms}$ to $150 \mathrm{~ms}$ thus the total display and ISI time was increased by $800 \mathrm{~ms}$. The total time for each trial was therefore $13,400 \mathrm{~ms}$. With 12 groups, counterbalancing procedures provided for each slide to be seen in three of the eight serial positions. The design and procedure was otherwise as specified in the General Method section.

\section{Results and Discussion}

Results of Experiment 2 are shown on Figure 3. The shape of the curves is similar to that produced in Experiment 1, however they are somewhat less variable. A comparison of Figures 3 and 2 shows that, as expected, the overall performance is higher for all conditions in Experiment 2 than it was in Experiment 1. In Experiment 2, the overall recognition probability was .46 whereas it was .34 in Experiment 1, however the difference is not statistically reliable, $t(155)=1.12, p=.14$. The figures show that the increment in performance is across all serial positions, including the recency portion of the curve.

As Table 1 shows, the model fits the data very well. The RMSE between observed and predicted values was 0.035 . The statistical test of the model against the data was not significant, $F(14,28)=0.044$. The best fitting $k$ value 


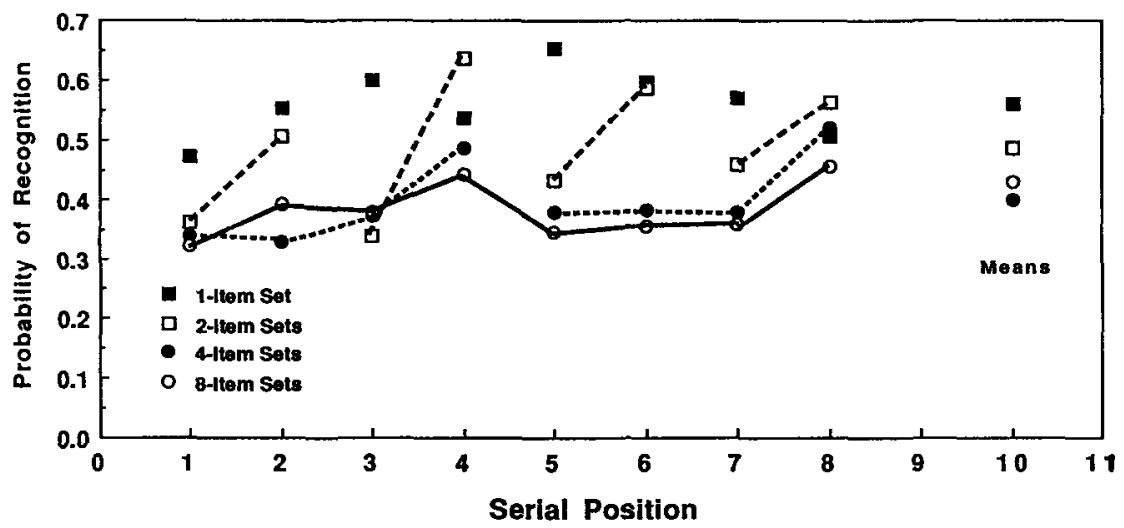

Fig. 3 Experiment 2: Increased Duration

for Experiment 2 was 0.88 which is identical to that obtained in Experiment 1 .

Again, there is a set-size effect such that the mean is higher for smaller sets, $F(3,150)=30.08$. Analyses by t-test indicated that the 2- and 4-scene sets were significantly different, $t(7)=3.19$ and the 1 - and 2-scene sets were marginally significantly different, $t(7)=1.52, p<.10$. As expected, the larger 4- and 8-scene sets were not significantly different, $t(7)=1.28$.

In the 4- and 8-scene sets, as predicted, the first position was not significantly different from the mean of the middle positions, $F(1,195)<1.0$, and $F(1,455)=1.14$ for the 4 - and 8 -scene sets respectively by planned comparisons. As in Experiment 1, the 1-scene set showed a significant negative primacy effect by a planned comparison, $F(1,455)=5.72, p<.05$, rather than the predicted flat function. The negative primacy effect in this condition may result from subjects' processing strategy. Their general strategy may be to expect an upcoming picture about half a second (400 ms) after the offset of the first picture. In three conditions, this expectation would be correct. In the 1-scene set condition, the result of this strategy may be less efficient use of available processing time $(1400 \mathrm{~ms})$ for the first scene and a negative primacy effect.

As the model predicts, there are significant recency effects in the 8-, 4-, and 2-scene sets. These were confirmed by planned comparisons of the last position against the middle positions with the data collapsed across sets in a trial, $F(1,455)=3.57 ; F(1,195)=14.51 ; F(1,65)=52.01$, for the 8-, 4-, and 2 -scene sets respectively.

The false-alarm rate was 0.287 . Standard errors of the mean were as follows: 0.026 for the 8-scene set; 0.018 for the 4 -scene sets; and 0.011 for the 2-scene sets.

As the model predicts, the increase in available processing time improved memory performance across all serial positions. Recency and set-size effects shown support the model's serial processing assumption. 


\section{Experiment 3: Reduced Luminance}

In Experiment 3 we reduced the luminance of the study pictures by $2 \log$ units, i.e., by $99 \%$, using neutral-density filters. Memory performance is predicted to be dependent on both the amount of conceptual processing time and the amount of perceptual information on which conceptual processing operates. Reducing the luminance of the pictures is expected to reduce the rate of perceptual information extraction (see Loftus, 1985) so that with a constant display time, the amount of perceptual information acquired from a dim picture would be less than that acquired from a normal-luminance picture. Because of the expected reduction in the rate of perceptual information acquisition, we permitted the perceptual information acquisition rate parameter, $c$, to vary in this experiment when fitting the model to the data. Thus the model has two free parameters in this experiment.

\section{Method}

Subjects and materials. Ninety-two subjects were run in 16 groups.

Design and procedure. As in Experiment 1 the stimulus duration was 50 ms which was followed by a 400-ms ISI so that the total time for each trial was $12,600 \mathrm{~ms}$. Filters were removed from the projectors at test so that the subjects saw the test pictures at normal luminance. As in Experiment 1, with 16 groups of subjects, counterbalancing of slides and serial positions resulted in each slide being seen in four of the eight serial positions. The design and procedure was otherwise as specified in the General Method section.

\section{RESULTS AND DISCUSSION}

The results of Experiment 3 are shown in Figure 4 and Table 1. A comparison of figures 2 and 4 show that, as expected, reducing the slide luminance resulted in a lower recognition probability. The overall mean recognition probability was .16 in Experiment 3 which is significantly lower than the value of .34 obtained in Experiment $1, t(181)=1.72, p<.05$.

The model provides a good fit to the data. The RMSE between observed and predicted values was 0.056 with the perceptual information processing rate parameter, $c$, allowed to vary to obtain the fit. The statistical test of the model against the data was not significant, $F(13,28)=0.068$. The best fitting $k$ value for Experiment 3 was 0.99 which is higher than the value of 0.88 obtained for both Experiments 1 and 2. Although the attention-switching parameter is close to unity, with reduced luminance, the rate of perceptual information acquisition for a stimulus is decreased. The best fitting value of $c$, the perceptual information acquisition rate, was 1.44 which is substantially lower than the value of 3.4 set for Experiments 1 and 2 and obtained for other normal-luminance stimuli. The model thus predicts lower recognition probabilities than in Experiments 1 and 2.

There is a set-size effect, $F(3,200)=22.37$. With the low recognition 


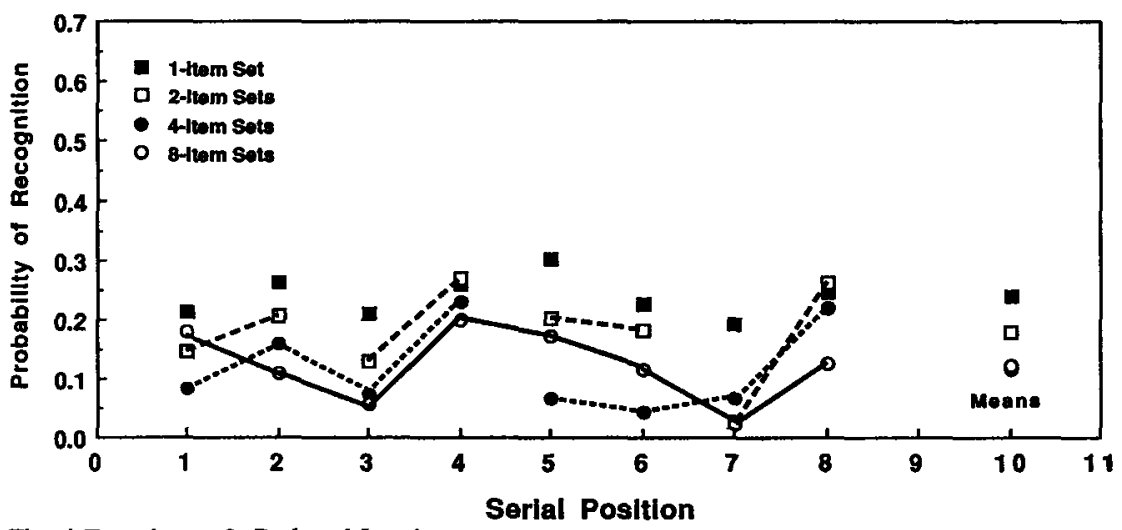

Fig. 4 Experiment 3: Reduced Luminance

probability, the model would predict a difference only between the 1-scene set and the other conditions. In the data, there are significant differences between the 1- and 2-scene sets, $t(7)=3.27$ and between the 2- and 4-scene sets, $t(7)$ $=2.74$, p. $<.05$. As can be seen on Figure 4, the means for the larger 4- and 8-scene sets do not differ, $t(7)=0.11$.

The model predicts recency effects for the 2-, 4-, and 8-scene sets. The recency effect is significant in the 2- and 4-scene sets but not in the 8-scene set by planned comparisons of the last position against the mean of the middle positions collapsed over sets in a trial, $F(1,91)=19.25, F(1,273)=18.13$, and $F(1,637)<1.0$ for the 2-, 4-, and 8-scene sets respectively.

The false-alarm rate was 0.345 . Standard errors of the mean were as follows: 0.022 for the 8-scene set; 0.015 for the 4-scene sets; and 0.011 for the 2-scene sets.

\section{General Discussion}

We carried out these experiments with the intention of determining whether visual serial position effects are like verbal serial position effects. It appears that there are some important differences. In this section, we identify the factors contributing to the differences, assess the fit of the model, and draw conclusions.

\section{EXPERIMENTAL DATA}

Typically, verbal serial position curves show a primacy effect, a flat asymptote, a recency effect and an effect of list length. Our model predicts a flat function at the beginning and middle serial positions, a one-item recency effect, and an effect of set size for small sets.

\section{FIRST POSITION}

In contrast to results with verbal stimuli, serial position curves generated from 
our experiments using naturalistic scenes consistently show a relatively flat function at the beginning position rather than the primacy effect expected with verbal stimuli. Typically, the primacy effect for verbal stimuli is attributed to differential rehearsal for the first item. That is, the subjects continue to rehearse the first items as they proceed through the list. Given a list of words, this strategy provides a intuitively plausible explanation and one that is consistent with the evidence (see Crowder, 1976, for a review). It is not apparent that such a rehearsal strategy would be effective or even possible with a sequence of naturalistic scenes. Rather, the probability of the first scene capturing attention will be equivalent to the probability of other scenes capturing attention. This lack of a primacy effect is indicative of processing differences between verbal stimuli and visual stimuli such as the scenes used in these experiments.

Results of our experiments are consistent with other experiments using visual stimuli which also show no primacy effect (Atkinson et al., 1964; Christie \& Phillips, 1979; Intraub \& Nicklos, 1981; Loftus, 1974; Potter \& Levy, 1969; Shaffer \& Shiffrin, 1972; Shiffrin, 1973).

\section{RECENCY EFFECT}

In verbal learning experiments, the recency effect is often explained as due to temporal discriminability at retrieval (Crowder, 1976). That is, items enter into a memory store and the last items entered are easiest to retrieve; memory performance is a function of ease of retrievability. In contrast to the retrieval-stage explanation given for the recency effect in verbal learning, in visual memory, the recency effect is caused at the encoding stage. It depends on the amount of available processing time for the final item relative to other set items (Hanna \& Loftus, 1992). Compared to verbal learning experiments, the recency effect is small. It is shown only if available processing time is limited and there is potential for conceptual masking of sequence items, i.e., in the 8-, 4-, and 2-scene sets. Under those conditions, because there is no subsequent scene to conceptually mask it, the last scene will be better remembered than the others. In the 1-scene sets, however, because available processing time is sufficient, i.e., there is a one-second post-set interval after each scene, there will be no conceptual masking of sequence scenes and no differential processing of the last scene, hence no recency effect. Any additional available time after the last scene will not be utilized. Thus, a one-item recency effect will be shown only when available processing time is limited; otherwise, there will be no recency effect.

\section{SET-SIZE EFFECT}

The set-size effect is analogous to the list-length effect predicted by verbal learning models. Memory performance will be a function of set size, smaller 
sets resulting in higher performance. The pattern of expected results is somewhat different from that expected in verbal learning experiments, however. In verbal learning experiments, the effect depends on the ratio of primacy and recency items to middle items. Both primacy and recency effects are large in verbal learning experiments, hence the list-length effect holds over relatively long list lengths. For visual stimuli, the set size effect is limited to relatively small sets for two reasons. Firstly, it depends on the ratio of only the last to other items, i.e., there is no predicted primacy effect. Secondly, the recency effect is limited to one item, so the ratio is smaller than it would be if the effect were a multiple-item effect.

\section{ASSESSMENT OF THE MODEL}

The revised model fits the data well in most respects. Generally the functions are relatively flat at the initial serial position and at the middle positions. Recency effects shown were consistent with the model: in the 8-, 4-, and 2-scene sets, additional processing time at the last serial position led to improved recognition performance for it. The model's predicted pattern of set-size effects only for relatively small sets also fit the observed pattern well. The fits were obtained with only one free parameter in Experiments 1 and 2 and two free parameters in Experiment 3.

According to the model, as the stimulus duration increases, the amount of perceptual and conceptual processing increases and the memory representation becomes more complete. Thus improved memory performance is predicted. Experiment 2 showed that increasing the amount of available conceptual processing time led to improved recognition performance.

With reduced luminance, the model predicts a decreased perceptual information acquisition rate and subsequent decrement in predicted recognition performance. Experiment 3 showed that decreasing the luminance of stimuli led to a decrement in recognition memory performance. The best fit of the exper-imental data to the model was obtained with a lower value of the perceptual information acquisition rate parameter than that set for Experiments 1 and 2.

\section{Conclusion}

Combined with other results from the picture-memory literature, the results led to conclusions about conceptual processing of visual information. The flat function at the initial serial position, the one-item recency effect, and the set-size effect for small sets result from processing which is qualitatively different from verbal processing.

The data are fit well by a model which assumes that processing of visual stimuli is a serial process, that the subject switches attention from one stimulus to the next and that the attention switch is complete. 
Preparation of this article was supported by a National Research Council postdoctoral fellowship to Aura Hanna tenured at the Ames Research Center, N.A.S.A., and an NIMH grant to Geoffrey Loftus.

We thank Bernice Laden, James Lundell, Susan Infield and Penny Yee for helpful suggestions on the research issues being investigated and David Kutz, Curtis Small, and Jon Brewster for assistance with running the subjects. Please send correspondence to: Aura Hanna, Virginia Commonwealth University, Psychology Department, Box 2018, Richmond, Virginia 23284.

\section{References}

Atkinson, R., Hansen, D., \& Bernbach, H. (1964). Short-term memory with young children. Psychonomic Science, 1, 255-256.

Bernbach, H. (1975). Rate of presentation in free recall: A problem for two-stage memory theories. Journal of Experimental Psychology: Human Learning and Memory, 104, 18-22.

Bjork, R., \& Whitten, W. (1974). Recency-sensitive retrieval processes in long-term free recall. Cognitive Psychology, 6, 173-189.

Christie, D., \& Phillips, W. (1979). Simple drawing and pattern completion techniques for studying visualization and long-term visual knowledge. Memory and Cognition, 7, 360-367.

Cohen, R., (1970). Recency effects in long-term recall and recognition. Journal of Verbal Learning and Verbal Behavior, 9, 672-678.

Crowder, R. (1976). Principles of learning and memory. New York: Wiley.

Gillund, G., \& Shiffrin, R. (1981). Free recall of complex pictures and abstract words. Journal of Verbal Learning and Verbal Behavior, 20, 575-592.

Glanzer, M., \& Cunitz, A. (1966). Two storage mechanisms in free recall. Journal of Verbal Learning and Verbal Behavior, 5, 351-360.

Glenberg, A. (1984). A retrieval account of the long-term modality effect. Journal of Experimental Psychology: Learning, Memory, and Cognition, 10, 16-31.

Goodwin, C. J. (1976). Changes in primacy and recency with practice in single-trial free recall. Journal of Verbal Learning and Verbal Behavior, I5, 119-132.

Hanna, A., \& Loftus, G. (1992). The effect of expectation and available processing time on recognition of sequences of naturalistic scenes. Bulletin of the Psychonomic Society, 30, 251-254.

Hines, D. (1975). Immediate and delayed recognition of sequentially presented random shapes. Journal of Experimental Psychology: Human Learning and Memory, 5, 634-639.

Hines, D., \& Smith, S. (1977). Recognition of random shapes followed at varying delays by attended or unattended shapes, digits, and line grids. Journal of Experimental Psychology: Human Learning and Memory, 3, 29-36.

Intraub, H. (1984). Conceptual masking: The effects of subsequent visual events on memory for pictures. Journal of Experimental Psychology: Learning, Memory, and Cognition, 10, 115-125. 
Intraub, H., \& Nicklos, S. (1981). The capacity of short-term memory for successively presented pictures. Paper presented at the APA annual convention, Los Angeles.

Loftus, G. (1974). Acquisition of information from rapidly-presented verbal and non-verbal stimuli. Memory and Cognition, 2, 545-548.

Loftus, G. (1985). Picture perception effects of luminance on available information and information-extraction rate. Memory and Cognition, 2, 545-548.

Loftus, G., \& Ginn, M. (1984). Perceptual and conceptual masking of pictures. Journal of Experimental Psychology: Learning, Memory and Cognition, 10, 435-441.

Loftus, G., Hanna, A., \& Lester, L. (1988). Conceptual masking: How one picture captures attention from another picture. Cognitive Psychology, 20, 237-282.

Loftus, G., \& Hogden, J. (1988). Picture perception: Information extraction and phenomenological appearance. In G. Bower (Ed.), The psychology of learning and motivation: Vol. 22. New York: Academic Press.

Loftus, G., Johnson, C., \& Shimamura, A. (1985). How much is an icon worth? Journal of Experimental Psychology: Human Learning and Memory, 5, 197-211.

Loftus, G., \& Loftus, E. (1988). Essence of statistics (2nd ed.). New York: Knopf.

Murdock, B. (1962). The serial position effect of free recall. Journal of Experimental Psychology, 64, 482-488.

Murdock, B. (1964). Proactive inhibition in short-term memory. Journal of Experimental Psychology, 2, 184-189.

Phillips, W., \& Christie, D. (1977). Components of visual memory. Quarterly Journal of Experimental Psychology, 29, 117-133.

Potter, M. (1976). Short-term conceptual memory for pictures. Journal of Experimental Psychology: Human Learning and Memory, 2, 509-522.

Potter, M., \& Levy, E. (1969). Recognition memory for a rapid sequence of pictures. Journal of Experimental Psychology, 81, 10-15.

Raaijmakers, J., \& Shiffrin, R. (1981). Order effects in recall. In J. Long \& A. Baddeley (Eds.), Attention and Performance IX. Hillsdale, N.J.: Erlbaum.

Raffel, G. (1936). Two determinants of the effect of primacy. American Journal of Psychology, 48, 654-657.

Shaffer, W., \& Shiffrin, R., (1972). Rehearsal and storage of visual information. Journal of Experimental Psychology, 92, 292-296.

Shiffrin, R. (1973). Visual free recall. Science, 180, 980-982.

Tversky, B., \& Sherman, T. (1975). Picture memory improves with longer on time and off time. Journal of Experimental Psychology: Human Learning and Memory, 104, 114-118. 


\section{Appendix}

We use the following notational conventions in the equations. For the independent variables:

$d$ is the stimulus exposure duration;

$a$ is the stimulus onset asynchrony (SOA).

For the dependent variables, the following notation is used:

$\mathrm{p}(\mathrm{L})$ is the probability that a picture is learned;

$\mathrm{p}(\mathrm{FA})$ is the observed false-alarm probability.

Acquired perceptual information. The proportion of total perceptual information that the observer has extracted, $I(d)$, is taken from the Loftus and Hogden (1988) perceptual information processing model. The general equation for $I(d)$ when a $d$-ms stimulus is followed by a perceptual mask at an SOA of $a \mathrm{~ms}$ is as follows:

$\mathrm{I}(\mathrm{d})=1.0-e^{-\mathrm{c}(d+w(1.0-\exp (-(-d-d) w)))}$

Eq. 1

where $w$ is the worth of any icon that follows the stimulus and $c$ is the perceptual information acquisition rate. We use the $c$ value of 3.4 estimated by Loftus et al. (1988). The model assumes that having an icon is equivalent, in terms of perceptual information acquisition, to extending the picture's physical duration, $d$, by $w$ ms. Loftus, Johnson, and Shimamura (1985) estimated the icon's worth to be $100 \mathrm{~ms}$. We use this estimate in the model. A special case of Equation 1 applies to the present experiments. When a mask is delayed by $300 \mathrm{~ms}$, i.e., when there is an icon, the term, $\exp (-(a-d) / w)$, in Equation 1 is approximately zero; it reduces to

$I(d)=1.0-e^{-c(d+J 00)}$

Memory performance. At any given time, conceptual processing is allocated to a single picture. Attention is switched to a picture, and away from the currently attended to picture, with probability $k$. The probability of learning a picture will be influenced by the attention-switching probability, $k$, the amount of acquired perceptual information, $\mathrm{I}(\mathrm{d})$, the rate of conceptual processing, $s$, and the amount of conceptual processing time determined by $a$, the SOA. Loftus et al. (1988) estimated the value of $s$, the conceptual information processing rate, to be 2.14 . We use this estimate in the model. The learning probability, $p(L)$, under various circumstances, is determined as follows:

0 for a picture that was never attended to; $\mathrm{p}(\mathrm{L})=[I(d)]\left(1.0-e^{-s a i)}\right.$ for a picture attended to for exactly $i$ trials;

Eq. 2 $I(d)$ for a picture being attended to during a post-set interval. 
Consider now the picture in serial position $n$ of a set $(n=1,2, \ldots, N)$. The learning probability, conditional on the picture's being attended to when it is presented, is the sum of two joint probabilities: the probability that the picture is attended to before the post-set interval and is learned, plus the probability that the picture is attended to during the post-set interval and is learned. The first term is the sum of $\mathrm{N}$-n separate terms, each representing the probability that the picture is attended to for exactly $i$ trials $(i=1,2, \ldots, N$-n) times the learning probability conditional on being attended to for $i$ trials. The values of the separate terms decrease as a function of the number of possible trials as the probability of an attention-switch to a subsequent picture increases. The second term represents the probability that the picture is attended to during the post-set interval. This will occur for a picture from which attention has not been captured. The value of this term increases as the end of the sequence nears. Thus, to obtain the learning probability for the picture in serial position $\mathrm{n}$, conditional on the picture's being attended to in the first place, we sum over $i$, the possible number of attended-to trials.

$\mathrm{p}($ Llattended to $)=\sum\left\{\left[(1-k)^{i-1} k\right]\left[I(d)\left(1.0-e^{-s a i}\right]\right\}+\left[(1-k)^{N \cdot n}\right][I(d)]\right.$

Eq. 3

The unconditional learning probability is the weighted sum of the learning probabilities given that the picture is or is not attended to begin with. Because the learning probability is zero if the picture is not attended to,

$\mathrm{p}(L)=x p($ Llattended to $)$

where $x=k$ for the pictures in the set $(n=1,2,3, \ldots, N)$. 